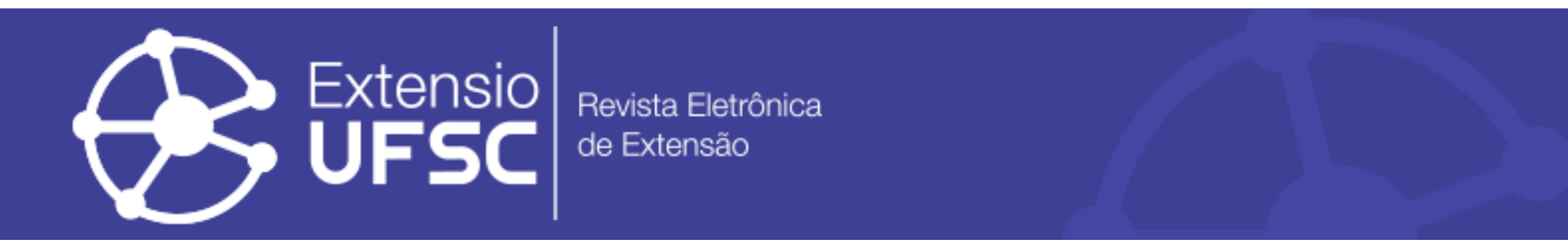

\title{
ABORDANDO HÁBITOS SAUDÁVEIS COM ESCOLARES: RELATO DE EXPERIÊNCIA
}

\author{
Raul Vinicius Eleutério \\ Universidade Federal de Santa Catarina \\ raul.eleuterio96@gmail.com \\ Luciara Fabiane Sebold \\ Universidade Federal de Santa Catarina \\ fabisebold@gmail.com
}

\author{
Bárbara Morh da Silveira \\ Universidade Federal de Santa Catarina \\ barbaramohrs@gmail.com \\ Juliana Simas Justino \\ Universidade Federal de Santa Catarina \\ jusimasj14@gmail.com
}

Palloma Caroline Guedes Oliveira

Universidade Federal de Santa Catarina pallominha_oliver@hotmail.com

\begin{abstract}
Resumo
Trata-se de um relato de experiência vivenciado por bolsistas de extensão do projeto "Desenvolvendo Hábitos Saudáveis junto aos alunos da rede básica de educação". Objetivo: desenvolver atividades de promoção da saúde com alunos através atividades lúdicas. Método: As atividades foram realizadas por graduandos do Curso de Enfermagem da UFSC com 60 alunos do $5^{\circ}$ ano de uma escola da rede básica estadual de Florianópolis. Resultados: através da realização deste projeto os graduandos do Curso de Enfermagem puderam vivenciar a criação de vínculos de confiança e fortalecimento na relação entre os profissionais da área da saúde com os estudantes e professores da escola. Conclusão: Permitindo assim, que os acadêmicos estejam aptos a planejar, organizar e atuar em atividades educacionais que favoreçam a promoção de saúde acerca de hábitos saudáveis em escolas. Período: segundo semestre de 2015.
\end{abstract}

Palavras-chave: Obesidade. Obesidade Infantil. Hábitos Alimentares.

\section{ADDRESSING HEALTHY HABITS TO SCHOOLS: EXPERIENCE REPORT}

\section{Abstract}

This is an experience report experienced by project extension fellows "Developing Healthy Habits to the students of basic education network." Objective of developing health promotion activities with students through recreational activities. The activities were carried out with 60 students of the 5th year of a school state basic network Florianópolis. Through the realization of this project the graduates of UFSC nursing course might experience creating bonds of trust and strengthening the relationship between health care professionals with students and school teachers. Thus allowing the students are able to plan, organize and perform in educational activities that encourage health promotion on healthy habits in schools.

Key words: Obesity. Child Obesity. Eating Habits.

\section{ABORDANDO HÁBITOS SALUDABLES CON ESCOLARES: RELATO DE EXPERIENCIA}

\section{Resumen}

Se trata de un relato de experiencia vivida por los becarios de extensión del proyecto "Desarrollo de hábitos saludables a los estudiantes de educación básica de la red." Objetivo de desarrollar actividades de promoción de la salud con los estudiantes a través de actividades recreativas. Las actividades se llevaron a cabo con 60 estudiantes del quinto año de una red básica estatal escuela Florianópolis. A través de la realización de este proyecto los graduados de curso de enfermería UFSC pueden experimentar la creación de lazos de confianza y el fortalecimiento de la relación entre profesionales de la salud con los estudiantes y profesores de la escuela. Por lo tanto permitiendo que los estudiantes sean capaces de planificar, organizar y llevar a cabo actividades educativas que fomenten la promoción de la salud sobre hábitos saludables en las escuelas.

Palabras clave: Obesidad. La Obesidad Infantil. Los Hábitos Alimenticios. 
Abordando hábitos saudáveis com escolares: relato de experiência

\section{INTRODUÇÃO}

Os hábitos saudáveis devem ser cultivados em todas as etapas da vida, ou seja, desde a gestação, passando pela infância, vida adulta e seguindo até o envelhecimento, sendo que a realização destes envolve, dentre outras coisas, a prática de atividades físicas e a manutenção de uma alimentação saudável. Dentro da Política de Promoção da saúde, homologada em 2006 e ratificada em 2014 pela Portaria no 2.446, de 2014, dentre outros, há dois temas prioritários, dentre eles destaca-se "Alimentação adequada e saudável" e, o outro, "Práticas corporais e atividades físicas" (BRASIL, 2014).

Para uma alimentação ser considerada saudável ela necessita seguir princípios como: Ser adequada em quantidade e qualidade, ou seja, devem-se oferecer de maneira equilibrada todos os nutrientes necessários para cada fase da vida; Ser variada de forma a facilitar a oferta de todos os nutrientes necessários ao organismo; Segura dos pontos de vista sanitário e genético; Garantida ao acesso físico e financeiro; Atrativa do ponto de vista sensorial; Respeito à cultura alimentar do indivíduo ou grupo a que se destina (BRASIL, 2006).

Com isso, sabe-se que uma alimentação inadequada e uma prática de atividade física reduzida podem levar a obesidade. Tendo isto em vista, ressaltasse a importância da estimulação de saudáveis desde a infância, pois elas podem auxiliar na prevenção do sobrepeso e a obesidade.

A obesidade é definida como o acúmulo anormal de gordura e apresenta um risco para a saúde (ORGANIZACIÓN MUNDIAL DE LA SALUD, 2017). Em consequência do excesso de peso, podem surgir algumas comorbidades como: doença renal, doenças cardiovasculares, diabetes Mellitus. Além disso, a obesidade pode causar incapacidade funcional, reduzir a qualidade de vida e aumentar a mortalidade (ABESO, 2016).

Segundo dados do Instituto Brasileiro de Geografia e Estatística (IBGE), entre os anos de 2008 e 2009, 21,7\% das crianças e jovens entre 10 e 19 anos do sexo masculino apresentavam excesso de peso, e 5,9\% já apresentavam obesidade. Já no sexo feminino, neste mesmo período, 19,4\% de meninas apresentavam excesso de peso e 4\% obesidade. A região sul, quando comparado a outras regiões do Brasil, é a que apresenta maior índice de excesso de peso, tanto no sexo masculino, quanto no sexo feminino, dentre os meninos dos 10 aos 19 anos (27,2\%), seguida pela região Sudeste $(24,6 \%)$, Centro-Oeste $(24,2 \%)$, Norte $(18,5 \%)$ e Nordeste $(16,1 \%)$. Já dentre as meninas dos 10 aos 19 anos, a região sul também apresenta maior índice de excesso de peso $(22,0 \%)$, seguida pela região Sudeste $(21 \%)$, Centro-Oeste $(20,1 \%)$, Nordeste $(17,1 \%)$ e Norte (16,4\%) (BRASIL, 2010). 
Abordando hábitos saudáveis com escolares: relato de experiência

A implementação da prática de hábitos saudáveis deve ser constante e diária. Nesta perspectiva, construiu-se o projeto de extensão intitulado "Desenvolvendo Hábitos Saudáveis junto aos alunos da rede básica de educação" com o objetivo de Desenvolver atividades de promoção da saúde e qualidade de vida com os alunos através atividades lúdicas de práticas de exercícios físicos vinculadas aos alimentos saudáveis.

\section{MATERIAIS E MÉTODOS}

Trata-se de um relato de experiência descrito a partir das atividades desenvolvidas durante a execução do projeto mencionado acima. A atividade foi desenvolvida pelos discentes de graduação em Enfermagem da quarta fase do curso, supervisionados pelo docente coordenador do projeto, com 60 crianças, entre 10 e 11 anos, do $5^{\circ}$ ano do ensino fundamental de uma escola da rede básica de educação, localizada na cidade de Florianópolis, Santa Catarina/Brasil.

Todo o processo, envolvendo a aproximação com a temática até o planejamento das atividades de intervenção, foi realizado ao longo do ano 2015, sendo que a execução da atividade na escola foi realizada nos meses de setembro e outubro do mesmo ano. Assim as atividades foram executadas em três etapas interdependentes e complementares descritas a seguir.

Etapa 1: Aproximação teórica da temática

Nesta etapa buscou-se na literatura o aprofundamento teórico das questões voltadas para os hábitos saudáveis, obesidade infantil e atividades lúdicas com escolares. A partir disso, percebeu-se que o embasamento teórico subsidiou as ações e atividades a serem desenvolvidas promovendo maior segurança para a formação do acadêmico de enfermagem no que se refere à temática e ao trabalho em escolas.

Etapa 2: Planejamento das atividades

Tendo em vista a importância da temática proposta e levando em consideração a faixa etária dos alunos envolvidos, procurou-se elaborar a atividade com enfoque lúdico, visando assim, a maior participação dos escolares na atividade.

Para finalizar o planejamento, entrou-se em contato com a escola e ficou acordado que as atividades seriam realizadas em um tempo de 45 minutos, em dois dias distintos, um com a turma do período vespertino, e no outro com a turma do período matutino.

Após diversas reuniões, foi decidido pela realização de um jogo de tabuleiro, objetivando a realização de atividades físicas e um diálogo descontraído a respeito de alimentação. 
Abordando hábitos saudáveis com escolares: relato de experiência

O tabuleiro proposto continha vinte e oito "casas" o qual as crianças ficavam em pé, sendo que suas divisões eram: três alimentos saudáveis (cenoura, maça e brócolis); duas atividades físicas (polichinelo e pula corda); três alimentos não saudáveis (frituras, refrigerantes e hambúrguer) e dois fast-food. As demais eram contadas como "casas em branco" e ficavam entre as casas onde havia os desenhos citados a cima.

Além disso, também foi elaborado um folder, com informações a respeito da prática de hábitos saudáveis e do consumo excessivo de sal, para que os escolares pudessem passar as informações obtidas na escola para seus responsáveis e demais familiares. Por fim, também foi realizada a elaboração de um questionário sobre os hábitos alimentares e de atividades físicas que seria respondido pelos alunos.

Ao final do planejamento, os acadêmicos puderam perceber a importância da realização do planejamento antes da realização da atividade propriamente dita, sendo que esta etapa é imprescindível para o sucesso da ação.

Etapa 3: Intervenção na realidade

Primeiramente o grupo de acadêmicos apresentava-se a turma e explicavam o objetivo da atividade e qual atividade seria realizada, após isto, entregavam o folder e o questionário. Então, a turma era dividida aleatoriamente em cinco equipes, sendo que cada um destas elegia um representante que ficaria responsável pelo grupo.

O aluno responsável ficaria, no primeiro momento, no tabuleiro, arremessava o dado e andaria a quantidade de passos indicado no mesmo. Caso parasse em uma casa que continha alimento saudável, seria premiado andando uma casa para frente. Já se parasse em uma casa representando atividade física, a sua equipe deveria realizá-la. Por fim, se parasse em uma casa que continha algum alimento não saudável, deveria voltar uma casa do tabuleiro. Ainda havia a possibilidade de parar em uma casa com figuras representando fast-food, caso isto ocorresse, o aluno ficaria uma rodada sem jogar. Para uma melhor compreensão, o esquema do tabuleiro pode ser visualizado nas figuras abaixo: 
Abordando hábitos saudáveis com escolares: relato de experiência

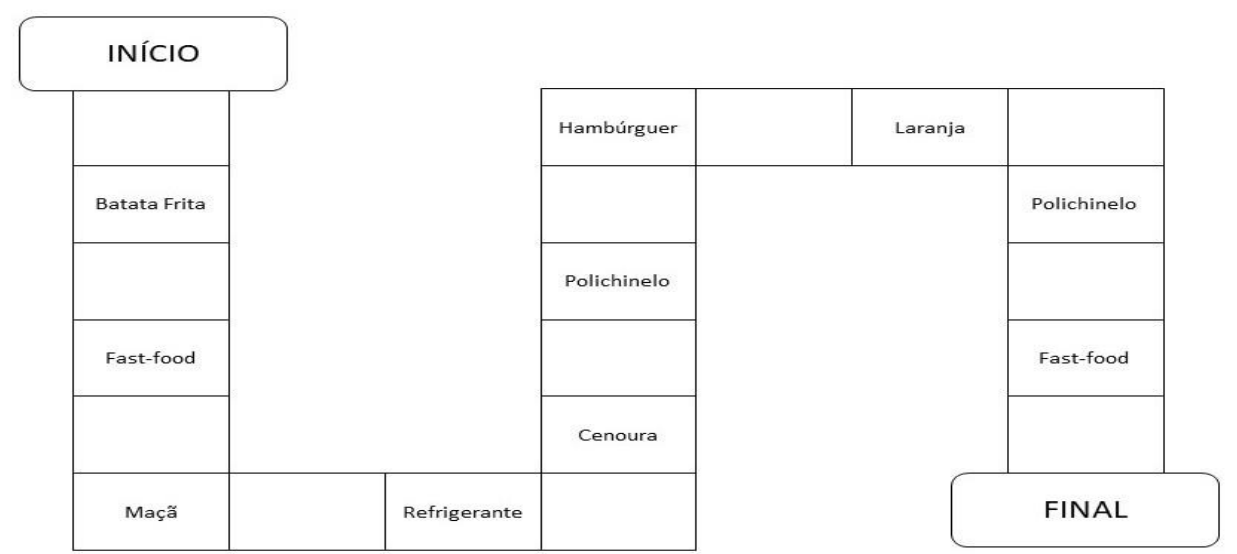

Figura 1. Tabuleiro

Fonte: elaborado pelos autores

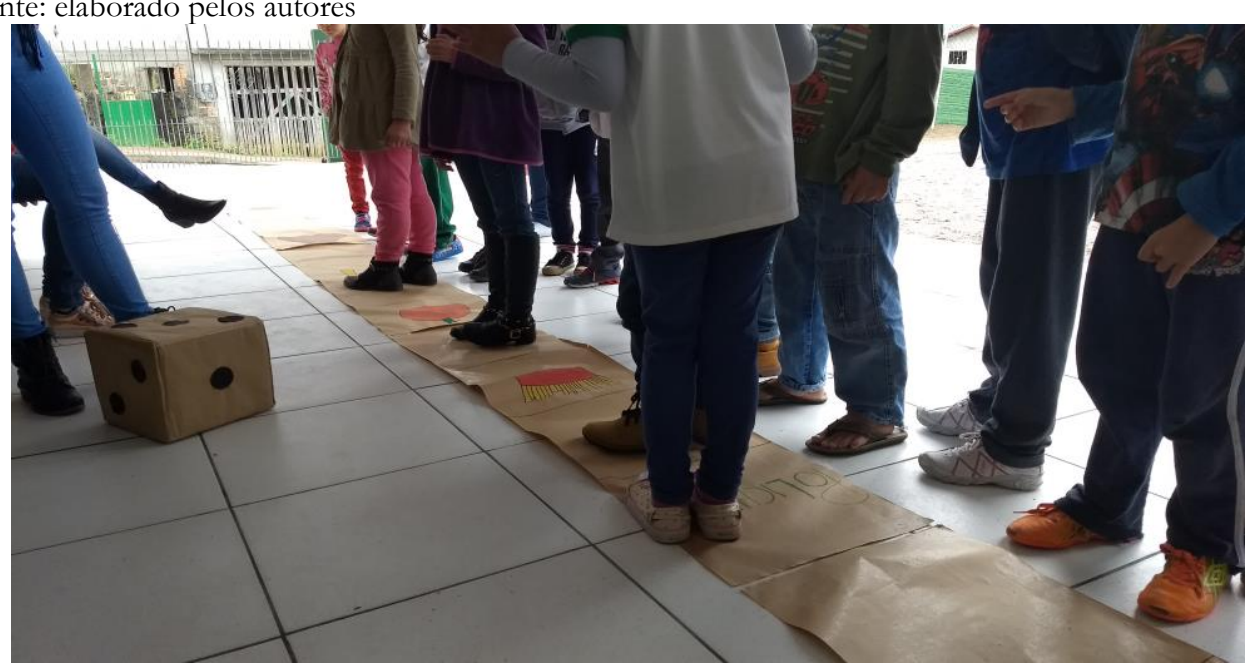

Figura 2. Atividade com o Tabuleiro

Fonte: Acervo Projeto 
Abordando hábitos saudáveis com escolares: relato de experiência

\section{RESULTADOS E ANÁLISES}

Durante a realização da atividade foi visível o envolvimento e interesse por parte dos escolares. Foi possível observar um conhecimento pré-existente a respeito de alguns alimentos saudáveis e não saudáveis, o que nos levou a refletir sobre o impacto desta atividade na vida cotidiana dos alunos.

Sabe-se que a o desenvolvimento do sobrepeso e obesidade na vida das pessoas é uma realidade inevitável, no entanto, existem estratégias educativas que podem minimizar sua influência negativa, como por exemplo, a utilização de estratégias de ensino alternativas podem auxiliar os alunos a propor formas de discussão, onde possam manifestar-se e também refletirem sobre suas vidas, redimensionamento os hábitos considerados não saudáveis e os substituindo por outros mais saudáveis (SALUM, 2015).

A escola é um local propicio para a disseminação de informações a respeito de hábitos saudáveis, entretanto configura-se como um grande o desafio a incorporação destes temas dentro do contexto escolar (BRASIL, 2006).

Outro aspecto importante a ser considerado é que a escola também pode ser um espaço de aprendizado para os acadêmicos de enfermagem, tendo em vista o papel do enfermeiro na orientação dos alunos e dos pais a respeito da obesidade e os agravos que podem ser decorrentes dela (NASCIMENTO, 2011).

O enfermeiro tem um importante papel na prevenção da obesidade infantil, portanto deve atender às necessidades de crianças com sobrepeso e obesidade também nas escolas. Os enfermeiros que atuam nestes ambientes podem auxiliar na promoção de uma alimentação saudável, realizar o acompanhamento estudantes, funcionários e famílias, com o intuito de identificar crianças em sobrepeso, por fim, pode também estimular um estilo de vida saudável, e atuar em programas que enfocam a prevenção da obesidade (WRIGHT, et al., 2013).

Vale ressaltar ainda que, as atividades desenvolvidas de ensino, pesquisa e extensão nos cursos auxiliam os acadêmicos expandir suas competências. A extensão tem importância fundamental na formação do futuro profissional, pois envolvem ao mesmo tempo alunos, professores, pesquisadores, e em especial, na enfermagem, pois o desenvolvimento de propostas de trabalho junto à educação fundamental nas instituições de ensino, pois fortalece o aprendizado dos acadêmicos de enfermagem. 
Abordando hábitos saudáveis com escolares: relato de experiência

Do ponto de vista do ensino na graduação em enfermagem, a incorporação de discussões com a temática da obesidade busca qualificar os discentes para atuarem como agentes promotores da saúde com foco na obesidade, na perspectiva da inclusão de espaços de discussão e reflexão sobre a temática e atuação junto à comunidade escolar.

A participação dos alunos de graduação em projetos de extensão tem grande repercussão em suas vidas acadêmicas, pois são nestes espaços comunitários que eles podem colocar em prática seus aprendizados e, acima de tudo acabam contribuindo para a transformação social, como no caso das atividades desenvolvidas neste projeto, que podem vir a ser um diferencial na vida destes escolares e suas famílias. As possibilidades de práticas educativas em saúde são determinantes na construção de um sistema de saúde mais integral, articulando as dimensões de cuidado, educação e gerenciamento relacionados a práticas de atenção à saúde, aspectos pelos quais contribuem significativamente na formação em Enfermagem (SALUM, 2015).

\section{CONSIDERAÇÕES FINAIS}

A inserção da temática obesidade desde a graduação é de suma importância para a formação do enfermeiro, tendo em vista que anualmente são desenvolvidas novas pesquisas e ocorre à descoberta de novas informações acerca da temática, os estudantes, em especial os da Enfermagem, precisam ser incentivados e aconselhados à pesquisa e a extensão.

Com a realização desta atividade, os graduandos do Curso de Enfermagem da UFSC puderam vivenciar a criação de vínculos de confiança e fortalecimento na relação entre os profissionais da área da saúde com os estudantes e professores da escola, permitindo assim, que os acadêmicos sejam aptos a planejar, organizar e atuar em atividades educacionais que favoreçam a promoção de saúde acerca de hábitos saudáveis em escolas.

Por fim, ressalta-se a importância da atuação do enfermeiro nos ambientes escolares, sendo que este pode ser um espaço de autonomia e de grandes contribuições na comunidade escolar, além de articular o ensino e o serviço da atenção básica.

\section{REFERÊNCIAS}

ABESO. Associação Brasileira para o Estudo da Obesidade e da Síndrome Metabólica. Diretrizes brasileiras de obesidade 2016 / ABESO - Associação Brasileira para Estudo da Obesidade e da Síndrome Metabólica. - 4.ed. - São Paulo, SP. 
Abordando hábitos saudáveis com escolares: relato de experiência

BRASIL. Ministério da Saúde. Secretaria de Vigilância em Saúde, Política nacional de promoção da saúde./ Ministério da Saúde, Secretária de Atenção à Saúde - Brasília, 2006.

BRASIL. Instituto Brasileiro de Geografia e Estatística - IBGE. Pesquisa de orçamentos familiares 2008-2009. Brasil, 2010. Disponível em: $<$ http://www.ibge.gov.br/home/presidencia/noticias/imprensa/ppts/0000000108.pdf $>$ Acesso em 31 maio 2017.

BRASIL. Ministério da Saúde. Secretaria de Vigilância à Saúde. Secretaria de Atenção à Saúde. Política Nacional de Promoção da Saúde: PNaPS : revisão da Portaria MS/GM no 687, de 30 de março de 2006 / Ministério da Saúde, Secretaria de Vigilância à Saúde, Secretaria de Atenção à Saúde. - Brasília : Ministério da Saúde, 2014. 32 p

NASCIMENTO, Amanda Moreira; MAGALHÃES, Marcília Concebida; PAES, Maione Silva Louzada. ENFERMEIRO E ESCOLA: UMA PARCERIA NA PREVENÇÃO E CONTROLE DA OBESIDADE INFANTIL. Revista Enfermagem Integrada, Ipatinga, v. 4, n. 1, p.742754, ago. 2011. Disponível em: < https://www.unilestemg.br/enfermagemintegrada/artigo/v4/10-enfermeiro-e-escola-umaparceria-na-prevencao-e-controle-da-obesidade-infantil.pdf $>$. Acesso em: 31 maio 2017.

ORGANIZACIÓN MUNDIAL DE LA SALUD. Estrategia mundial sobre régimen alimentario, actividad física y salud. Disponível em: http://www.who.int/dietphysicalactivity/childhood what/es/>. Acesso em: 31 maio 2017.

SALUM, Gabriel de Barros; MONTEIRO, Luciana Alves Silveira. Educação em saúde para adolescentes na escola: um relato de experiência. Rev Min Enferm, Belo Horizonte, v. 19, n. 2, p.252-257, jun. 2015. Disponível em: < http://www.reme.org.br/artigo/detalhes/1019>. Acesso em: 31 maio 2017.

WRIGHT, Kynna et al. Impact of a nurse-directed, coordinated school health program to enhance physical activity behaviors and reduce body mass index among minority children. Int J Nurs Stud, Online, v. 20, n. 5, p.727-737, jun. 2013. Disponível em: < http://www.journalofnursingstudies.com/article/S0020-7489(12)00298-2/ fulltext?cc $={ }_{y}=>$. Acesso em: 31 maio 2017.

Recebido em: 09/10/2017

Aceito em: 04/07/2018 